\title{
The Contribution of Alcohol Consumption to Overall Cancer Incidence in the West- ern World: A Meta-Analysis
}

Hendriks $\mathrm{HFJ}^{*}{ }^{*}$, and Calame $\mathrm{W}^{2}$

${ }^{1}$ Hendriks Nutrition Support for Business, Zeist, Netherlands

${ }^{2}$ StatistiCal BV, Wassenaar, Netherlands

${ }^{*}$ Corresponding author: Hendriks HFJ, Hendriks Nutrition Support for Business, Laan van Cattenbroeck 70 3703 BP Zeist, Zeist, Netherlands, Tel: +31651010454, E-mail: henk.hendriks5@gmail.com

Citation: Hendriks HFJ, Calame W (2018) The Contribution of Alcohol Consumption to Overall Cancer Incidence in the Western World: A Meta-Analysis. J Nutr Health Sci 5(3): 308. doi: 10.15744/2393-9060.5.308

Received Date: July 12, 2018 Accepted Date: September 12, 2018 Published Date: September 14, 2018

\begin{abstract}
Background: The effect of alcohol consumption on overall cancer incidence is not clear. The aim of the paper is to estimate the impact of alcohol consumption on risk distribution of the 20 most common cancer types among men and women in the Western world.

Methods: A meta-analysis of relative risks for the 20 most common cancer types potentially associated with alcohol consumption in the Western world was conducted based on the most recent cancer specific meta-analyses. Cancer risks were compared between men and women applying a sigmoidal dose response model.

Results: Drinking 2.5-14.9 g alcohol/day was associated with a small decrease in overall cancer incidence: 0.977 for men and 0.974 for women; followed by a small increase in the 15-29.9 g/day category: 1.029 for men and 1.077 for women. Further increases were observed in the 30-60 g/day category indicating a 5 and 10\% increase in overall cancer risk for men and women, respectively. Women appeared to be more sensitive: the alcohol consumption level to acquire overall risk increase is $22 \mathrm{~g} /$ day for women and $46 \mathrm{~g} / \mathrm{day}$ for men.

Conclusion: Moderate alcohol consumption (up to $15 \mathrm{~g} /$ day) is not associated with the incidence of the 20 most common cancer types in the Western world in contrast to higher consumption.
\end{abstract}

Keywords: Alcohol Consumption; Cancer Risk; Meta-Analyses

\section{Introduction}

Cancer is a leading cause of death in the Western world. The overall cancer death rate, however, has started to decline since the early 1990s [1,2]. Apart from significant progress in the treatment of various cancers, more attention is now focused on cancer prevention.

Many factors affect cancer incidence. Important risk factors most of which are lifestyle factors are: smoking, obesity, infections, physical inactivity, diet, occupational hazards, alcohol, reproductive factors, UV light/radiation, environmental pollutants and prescribed drugs. Their contribution to cancer incidence is considered to be relatively large, for smoking (about $30 \%$ ), obesity (about 20\%) and infections (about 15\%) [3,4]. Physical inactivity, diet and occupational hazards contribute for a smaller percentage of about 5\% each. The remainder risk factors contribute even less, with alcohol's contribution being estimated at about 3\% [3].

Heavy alcohol consumption has been linked to increased risk of several types of cancer including cancer of the female breast, male prostate, colorectal cancer, cancers of the upper aero-digestive tract and some others [11]. The overall effect of moderate and light alcohol consumption on cancer incidence is less clear [6,7]. Whereas some authors indicated a minimally increased or no increased risk of overall cancer others have suggested that even light and moderate drinking significantly contribute to an increased overall cancer risk [8-10].

Overall cancer risk is difficult to evaluate since cancer consists of various diseases, each bearing specific characteristics. Also, the association of each type of cancer varies considerably with alcohol consumption: some cancers are positively associated; others are negatively associated or not associated with alcohol consumption at all. Also, dose dependency, threshold level, age and gender may affect the association. Moreover, when the risk factor alcohol is considered for its contribution to cancer incidence, usually the positively associated cancer types are included only [11]. In this paper, the 20 most common cancer types were used to study the potential impact of alcohol consumption on cancer incidence, irrespective of their association with alcohol. 
In this paper, the associations between alcohol consumption and cancer risk of the 20 most common cancer types in both men and women in the Western world are described. Specifically, the relative importance of light and moderate alcohol consumption versus heavy drinking is discussed. Data published in meta-analyses of Western world epidemiological studies were used. The twenty most frequently occurring cancers in Europe were used as a representative of the Western world accounting for more than $80 \%$ of all cancers that have been researched for their association with alcohol consumption in a high-quality recent meta-analysis [12]. Moreover, a sigmoidal dose response model is presented to quantify the association between alcohol intake and cancer incidence risk in both men and women.

\section{Methods}

\section{Study Design}

A systematic literature search was conducted to evaluate the association between alcohol consumption and overall cancer incidence. To that purpose the twenty most common cancer types in Europe were selected as published in http://eco.iaarc.fr/ eucan/Cancer.aspx?Cancer, which contained data from 2012 onwards (Table 1). The cancer types identified were: female breast, prostate, colorectal, lung, bladder, renal, gastric, ovarian, non-Hodgkin lymphoma, skin, pancreatic, leukemia, liver, esophageal, larynx, brain glioma, multiple myeloma, gall bladder, thyroid and Hodgkin lymphoma. Some cancer types considered not to be associated with alcohol consumption were also included, to assure coverage of the most common types of cancers in the Western world. In contrast, others considered to be strongly associated with alcohol consumption, such as cancers of the oral cavity and pharynx, were not included because these cancers did not belong to the 20 most common cancers in Europe. Gender differences in cancer incidence were assessed via Chi-square or Fisher-exact analysis with one degree of freedom, depending on the level of expectation [13]. Since the overall cancer rate consisted of those of various types of cancers, to evaluate a gender difference 16 degrees of freedom were applied, not taking into account the gender-specific ones. A p-value below 0.05 was considered to demonstrate a statistically significant difference.

\begin{tabular}{|c|c|c|c|c|c|}
\hline & Cancer type & $\begin{array}{l}\text { Age standardized rate } \\
\text { per } 100,000 \text { men }\end{array}$ & $\begin{array}{c}\text { Percentage contribution } \\
\text { in men }\end{array}$ & $\begin{array}{l}\text { Age standardized rate } \\
\text { per } 100,000 \text { women }\end{array}$ & $\begin{array}{l}\text { Percentage contribution in } \\
\text { women }\end{array}$ \\
\hline 1 & Breast cancer* & 0 & 0 & 106.6 & 39.2 \\
\hline 2 & Prostate cancer ${ }^{*}$ & 105.5 & 26.8 & - & - \\
\hline 3 & Colorectal cancer ${ }^{*}$ & 59 & 15.0 & 36.1 & 13.3 \\
\hline 4 & Lung cancer* & 66.3 & 16.8 & 26.1 & 9.6 \\
\hline 5 & Bladder cancer ${ }^{*}$ & 29.1 & 7.4 & 6.1 & 2.2 \\
\hline 6 & Renal cancer & 17.4 & 4.4 & 8 & 2.4 \\
\hline 7 & Gastric cancer & 15.2 & 3.9 & 7.1 & 2.6 \\
\hline 8 & Ovarian cancer* & - & - & 12.6 & 4.6 \\
\hline 9 & $\begin{array}{l}\text { Non Hodgkin lym- } \\
\text { phoma }\end{array}$ & 13.8 & 3.5 & 9.6 & 3.5 \\
\hline 10 & Skin cancer & 13.2 & 3.4 & 13.1 & 4.8 \\
\hline 11 & Pancreatic cancer & 12.2 & 3.1 & 9 & 3.3 \\
\hline 12 & Leukemia & 11.8 & 3.0 & 7.1 & 2.6 \\
\hline 13 & Liver cancer & 11.1 & 2.8 & 3.6 & 1.3 \\
\hline 14 & Esophageal cancer & 8.4 & 2.1 & 2 & 0.7 \\
\hline 15 & Larynx cancer* & 8.3 & 2.1 & 0.9 & 0.3 \\
\hline 16 & Glioma, brain cancer & 8.1 & 2.1 & 5.9 & 2.2 \\
\hline 17 & Multiple myeloma & 5.5 & 1.4 & 3.7 & 1.4 \\
\hline 18 & Gall bladder cancer & 2.8 & 0.7 & 3.1 & 1.1 \\
\hline 19 & Thyroid cancer & 3.5 & 0.9 & 9.3 & 3.4 \\
\hline \multirow[t]{2}{*}{20} & Hodgkin lymphoma & 2.6 & 0.7 & 2.1 & 0.8 \\
\hline & Total & 393.8 & 100.1 & 272 & 99.3 \\
\hline
\end{tabular}

Table 1: Incidence of twenty most common cancer types possibly associated with alcohol drinking in men and women of the European Union^ $\wedge$ Data obtained from database: http://eco.iarc.fr/eucan/Cancer.aspx?Cancer.

* significant difference between men and women was assessed by Chi-square or Fisher exact with 1 degree of freedom applying a p-value below 0.05 , except for total cancer (19 degrees of freedom).

\section{Literature Search}

Publications were identified in June 2017 searching PubMed bibliographic databases for publications in English using the following keywords: cancer, alcohol and meta-analysis. These keywords were combined with the specific cancer type (e.g. breast) selecting 
further with the options 'review' as articles type and ' 10 years' for publication dates. Completeness was verified by a hand search on the most relevant journals of epidemiology and medicine and by comparing our search with that of general reviews and meta-analyses published on this topic. This search yielded 479 publications. Further selection criteria included: meta-analysis, meta-analysis on Western countries mainly ( $>80 \%$ of all persons included), meta-analysis with the highest number of studies and meta-analysis describing a dose-effect relation using relevant drinking categories. Finally the inclusion criterion 'most recent' was applied. Life time alcohol intake data were always preferred to baseline alcohol intake data in order to obtain the highest sensitivity in the outcome of the respective meta-analyses. From the 479 meta-analysis initially identified 34 meta-analyses were considered for use in this paper, finally including 20 papers fulfilling all selection criteria (Figure 1).

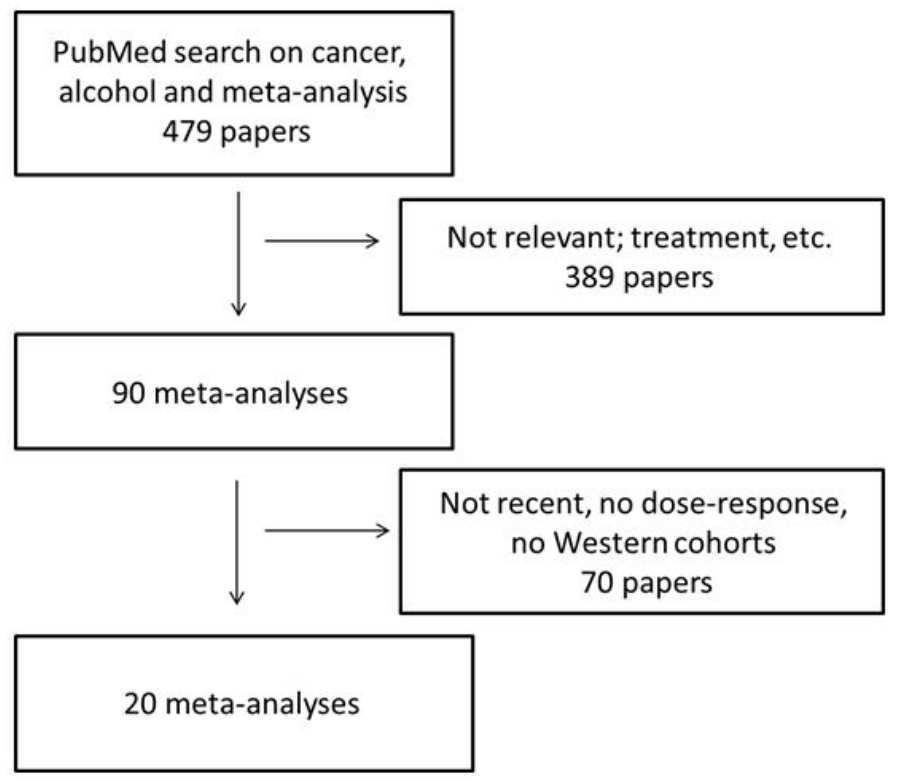

Figure 1: Schematic overview of search results

Associations expressed as hazard ratio, odds ratio and relative risk were treated as if they were all representing risk more or less the same way. Additionally, information from fully adjusted models was used when various models were presented in the metaanalyses selected. Importantly, meta-analyses containing Western cohorts mainly, were studied because associations for Eastern countries appeared to differ substantially for some cancers [14]. This was specifically the case for liver cancer for which there appeared to be no meta-analysis available that only comprised European and American cohorts. In general, the highest liver cancer incidences are found in Eastern and Southeastern Asia and parts of Africa among both sexes [15]. Therefore, one of the most recent meta-analyses comprising only about 50\% Asian studies was used [16].

\section{Alcohol Drinking Categories}

Intake was reported in grams of alcohol/day. Alcohol drinking categories were subdivided as follows: a non-drinking/occasional drinking control category of 0-2.5 $\mathrm{g}$ alcohol/day, 2.5-14.9 g alcohol/day, 15-29.9 $\mathrm{g}$ alcohol/day, 30-60 $\mathrm{g}$ alcohol/day and more than $60 \mathrm{~g}$ alcohol/day. The non-drinking / occasional drinking control group was chosen to minimize bias due to previous disease (sick quitters) and other confounders. For translation into common practice, a standard glass of alcoholic beverage was defined as 250 $\mathrm{ml}$ of beer, $100 \mathrm{ml}$ of wine and $35 \mathrm{ml}$ of spirits. This means that each standard glass alcoholic beverage contains about $10 \mathrm{~g}$ alcohol, which is the Dutch measure of a standard drink.

\section{Estimation of Overall Cancer Risk}

The overall contribution of alcohol consumption to cancer risk was estimated by totaling all available separate associations corrected for their relative contribution to overall cancer incidence. Overall incidence was estimated by multiplying the relative risk described for a specific cancer type in a specific drinking category with the relative incidence of that cancer type in the population [17]. Gender specificity was taken into account using both the gender specific alcohol related associations and the gender specific cancer type incidences.

\section{Modelling Cancer Risk}

Various models (such as polynomial and pseudo-Hill) were evaluated to analyze the association between alcohol consumption and cancer risk. A three-parameter logistic equation via a sigmoidal dose response (Hill) approach provided the best fit and the highest coverage of explained variation, as established via $\mathrm{R}^{2}[18]$. The various alcohol consumption categories were transformed to values being the average of the minimum and maximum value of that category. For the highest drinking category (more than $60 \mathrm{~g}$ alcohol/day), the value was set at $75 \mathrm{~g}$ alcohol/day using the same increase from the minimum value used for the previous 
category (30 to 60 with a mean of 45). Increasing or decreasing this mean value of the highest category did not substantially affect the outcome of the model.

The overall formula of the sigmoidal dose response model was:

$$
Y=\text { minimum }+\frac{(\text { maximum }- \text { minimum })}{\left(1+10^{(\operatorname{logEC50-x)})}\right.}
$$

in which:

Y: risk contribution per gender

$\mathrm{X}$ : mean of alcohol consumption category

logEC50: value at which $50 \%$ of the maximum effect is obtained

To compare the outcome of the impact by alcohol consumption on cancer risk between the various cancer types, the logEC50 value was used representing the inflection point of the model curve. The logEC50 value represents the dose at which $50 \%$ of the maximum effect has been reached. Since the effect could be an increase or a decrease, the direction of impact at logEC50 values is indicated as well. The sigmoidal approach was not only used for the various types of cancer but also for overall cancer risk and for comparing men and women. However, in some cancers no asymptotic level existed in risk in relation to alcohol consumption level. A parabolic function better reflected the association in those cases; a logEC50 value could, however, not be calculated.

\section{Results}

Incidences of the twenty most abundant cancer types in Europe are specified in Table 1, expressed as both the age standardized rate per 100,000 as well as the percentage contribution. Incidences are given for men and women separately.

Breast cancer and prostate cancer are the most abundant cancer types for women and men, respectively; both cancer types have been reported to be positively associated with alcohol drinking. From this table, it becomes clear that the following cancers were more observed in men than in women: colorectal, lung, bladder, esophageal, and larynx as demonstrated via Chi-square analysis. Overall the total incidence of the most commonly acquired cancers was not significantly higher in men than in women.

Seven and thirteen cancer types contributed less than an age-standardized rate of 10 per 100,000 for men and women, respectively.

\begin{tabular}{|c|c|c|c|c|c|c|c|c|c|c|c|}
\hline & & \multicolumn{5}{|c|}{ Relative Risk for Men } & \multicolumn{5}{|c|}{ Relative Risk for Women } \\
\hline & Alcohol (g/day) & $0-2.5$ & 2.5-14.9 & 15-29.9 & $30-60$ & $>60$ & $0-2.5$ & 2.5-14.9 & $15-29.9$ & $30-60$ & $>60$ \\
\hline & Cancer type & & & & & & & & & & \\
\hline 1 & Breast cancer(21) & 1 & 1 & 1 & 1 & 1 & 1 & 1.02 & 1.20 & 1.20 & 1.15 \\
\hline 2 & Prostate cancer(16) & 1 & 1.04 & 1.06 & 1.06 & 1.04 & 1 & 1 & 1 & 1 & 1 \\
\hline 3 & Colorectal cancer(36) & 1 & 1.06 & 1.13 & 1.27 & 1.35 & 1 & 1.06 & 1.13 & 1.27 & 1.35 \\
\hline 4 & Lung cancer(23) & 1 & 0.80 & 0.81 & 0.68 & 0.91 & 1 & 0.80 & 0.81 & 0.68 & 0.91 \\
\hline 5 & Bladder cancer(37) & 1 & 1 & 0.98 & 0.97 & 0.97 & 1 & 1 & 0.98 & 0.97 & 0.97 \\
\hline 6 & Renal cancer(38) & 1 & 0.85 & 0.73 & 0.66 & 0.60 & 1 & 0.85 & 0.68 & 0.54 & 0.54 \\
\hline 7 & Gastric cancer(39) & 1 & 1.03 & 1.13 & 1.17 & 1.12 & 1 & 1.03 & 1.13 & 1.17 & 1.12 \\
\hline 8 & Ovarian cancer(40) & 1 & 1 & 1 & 1 & 1 & 1 & 1.02 & 1.18 & 1.13 & 1.13 \\
\hline 9 & Non-Hodgkin lymphoma(41) & 1 & 0.88 & 0.87 & 0.87 & 0.84 & 1 & 0.88 & 0.87 & 0.87 & 0.84 \\
\hline 10 & Skin cancer(42) & 1 & 1.11 & 1.25 & 1.55 & 1.55 & 1 & 1.11 & 1.25 & 1.55 & 1.55 \\
\hline 11 & Pancreatic cancer(43) & 1 & 0.98 & 0.93 & 1.18 & 1.18 & 1 & 0.97 & 0.93 & 1.07 & 1.07 \\
\hline 12 & Leukemia(44) & 1 & 0.88 & 0.90 & 0.97 & 0.97 & 1 & 0.88 & 0.90 & 0.97 & 0.97 \\
\hline 13 & Liver cancer(16) & 1 & 1.05 & 1.08 & 1.08 & 1.59 & 1 & 0.81 & 1.24 & 1.24 & 3.89 \\
\hline 14 & Esophageal cancer(45) & 1 & 1.17 & 2.34 & 2.34 & 5.73 & 1 & 1.17 & 2.34 & 2.34 & 5.73 \\
\hline 15 & Larynx cancer(46) & 1 & 0.88 & 1.47 & 1.47 & 2.62 & 1 & 0.88 & 1.47 & 1.47 & 2.62 \\
\hline 16 & Glioma, brain cancer $(47,48)$ & 1 & 1 & 1 & 1 & 1 & 1 & 1 & 1 & 1 & 1 \\
\hline 17 & Multiple myeloma(49) & 1 & 0.96 & 0.89 & 0.85 & 0.98 & 1 & 0.96 & 0.89 & 0.85 & 0.98 \\
\hline 18 & Gall bladder cancer & 1 & 1 & 1 & 1 & 1 & 1 & 1 & 1 & 1 & 1 \\
\hline 19 & Thyroid cancer(50) & 1 & 0.83 & 0.75 & 0.80 & 0.80 & 1 & 0.80 & 0.73 & 0.71 & 0.80 \\
\hline 20 & Hodgkin lymphoma(51) & 1 & 0.71 & 0.73 & 0.73 & 0.74 & 1 & 0.71 & 0.73 & 0.73 & 0.74 \\
\hline
\end{tabular}


The relative risks for all twenty cancer types as a function of alcohol consumption are specified in Table 2 for men and women separately. Breast cancer risk was previously estimated to increase by about 10\% per $10 \mathrm{~g}$ alcohol [19], but more recent studies estimated these percentages to be somewhat lower [20,21]. An abundant cancer type, positively associated with alcohol consumption in both men and women, colorectal cancer, is increasing up to relatively high incidences at high consumption levels. Another high incidence cancer, lung cancer, on the other hand, is negatively associated with alcohol consumption in both genders. Bladder cancer, yet another high incidence type of cancer, appears to have no clear association with alcohol consumption.

Some cancer types with a lower incidence, defined as an age-standardized rate between 10 and 20 per 100,000 (e.g. renal and non-Hodgkin lymphoma) are negatively associated with alcohol consumption whereas others (e.g. pancreatic and liver cancer) are positively associated.

Liver cancer seems to occur more frequently in Eastern countries than in countries in the Western world [22]. The study by Bagnardi et al. used in this paper, however, did not observe heterogeneity based on geographical area using studies form both the Western and Eastern countries in a 50:50 ratio [16].

Risk contribution for twenty cancer types possibly associated with alcohol drinking in the Western World is specified in Table 3 for both men and women per alcohol consumption category.

Obviously, gender, as addressed via Chi-square evaluation (Table 1), affected the risk distribution for various gender specific cancers, like breast, prostate and ovarian, but gender differences were also observed for other cancers, namely renal, pancreatic, liver and thyroid cancer.

These contributions were combined to estimate the overall effect of alcohol consumption on the risk of acquiring cancer. The total, specified at the bottom of Table 3, was 1 for the non-drinkers and very light drinkers $(0-2.5 \mathrm{~g}$ alcohol/day). For the alcohol consumption category of 2.5-14.9 g alcohol/day the overall cancer risk was estimated at 0.98 for men and 0.97 for women, for the alcohol consumption category of 15-29.9 g of alcohol/day the overall cancer risk was estimated at 1.03 and 1.08 for men and women, respectively. Further increases were observed in the 30-60 g alcohol/day for men at 1.05 and for women at 1.10 indicating a 5 and $10 \%$ increase in overall cancer risk for men and women respectively as compared to the non-drinkers and very light drinkers.

\begin{tabular}{|c|c|c|c|c|c|c|c|c|c|c|c|c|c|}
\hline & & & \multicolumn{5}{|c|}{ Risk contribution for men } & & \multicolumn{5}{|c|}{ Risk contribution for women } \\
\hline & Alcohol (g/day) & & $0-2.5$ & 2.5-14.9 & $15-29.9$ & $30-60$ & $>60$ & & $0-2.5$ & $\begin{array}{l}2.5- \\
14.9\end{array}$ & $\begin{array}{r}15- \\
29.9\end{array}$ & $30-60$ & $>60$ \\
\hline & Cancer type & Contr ${ }^{*}$ & & & & & & Contr ${ }^{*}$ & & & & & \\
\hline 1 & Breast cancer & $\mathbf{0}$ & 0.000 & 0.000 & 0.000 & 0.000 & 0.000 & 39.2 & 0.392 & 0.400 & 0.470 & 0.470 & 0.451 \\
\hline 2 & Prostate cancer & 26.8 & 0.268 & 0.279 & 0.284 & 0.284 & 0.279 & $\mathbf{0}$ & 0.000 & 0.000 & 0.000 & 0.000 & 0.000 \\
\hline 3 & Colorectal cancer & 15.0 & 0.150 & 0.159 & 0.170 & 0.191 & 0.203 & 13.3 & 0.133 & 0.141 & 0.150 & 0.169 & 0.180 \\
\hline 4 & Lung cancer & 16.8 & 0.168 & 0.134 & 0.136 & 0.114 & 0.153 & 9.6 & 0.096 & 0.077 & 0.078 & 0.065 & 0.087 \\
\hline 5 & Bladder cancer & 7.4 & 0.074 & 0.074 & 0.073 & 0.072 & 0.072 & 2.2 & 0.022 & 0.022 & 0.022 & 0.021 & 0.021 \\
\hline 6 & Renal cancer & 4.4 & 0.044 & 0.037 & 0.032 & 0.029 & 0.026 & 2.4 & 0.024 & 0.020 & 0.016 & 0.013 & 0.013 \\
\hline 7 & Gastric cancer & 3.9 & 0.039 & 0.042 & 0.044 & 0.046 & 0.047 & 2.6 & 0.026 & 0.028 & 0.029 & 0.031 & 0.031 \\
\hline 8 & Ovarian cancer & $\mathbf{0}$ & 0.000 & 0.000 & 0.000 & 0.000 & 0.000 & 4.6 & 0.046 & 0.047 & 0.054 & 0.052 & 0.052 \\
\hline 9 & $\begin{array}{c}\text { Non Hodgkin lym- } \\
\text { phoma }\end{array}$ & 3.5 & 0.035 & 0.031 & 0.030 & 0.030 & 0.029 & 3.5 & 0.035 & 0.031 & 0.030 & 0.030 & 0.029 \\
\hline 10 & Skin cancer & 3.4 & 0.034 & 0.038 & 0.043 & 0.053 & 0.053 & 4.8 & 0.048 & 0.053 & 0.060 & 0.074 & 0.074 \\
\hline 11 & Pancreatic cancer & 3.1 & 0.031 & 0.030 & 0.029 & 0.037 & 0.037 & 3.3 & 0.033 & 0.032 & 0.031 & 0.035 & 0.035 \\
\hline 12 & Leukemia & 3.0 & 0.030 & 0.026 & 0.027 & 0.029 & 0.029 & 2.6 & 0.026 & 0.023 & 0.023 & 0.025 & 0.025 \\
\hline 13 & Liver cancer & 2.8 & 0.028 & 0.029 & 0.030 & 0.030 & 0.045 & 1.3 & 0.013 & 0.011 & 0.016 & 0.016 & 0.051 \\
\hline 14 & Esophageal cancer & 2.1 & 0.021 & 0.025 & 0.049 & 0.049 & 0.120 & 0.7 & 0.007 & 0.008 & 0.016 & 0.016 & 0.040 \\
\hline 15 & Larynx cancer & 2.1 & 0.021 & 0.018 & 0.031 & 0.031 & 0.055 & 0.3 & 0.003 & 0.003 & 0.004 & 0.004 & 0.008 \\
\hline 16 & Glioma, brain cancer & 2.1 & 0.021 & 0.021 & 0.021 & 0.021 & 0.021 & 2.2 & 0.022 & 0.022 & 0.022 & 0.022 & 0.022 \\
\hline 17 & Multiple Myeloma & 1.4 & 0.014 & 0.013 & 0.012 & 0.012 & 0.014 & 1.4 & 0.014 & 0.013 & 0.012 & 0.012 & 0.014 \\
\hline 18 & Gall bladder cancer & 0.7 & 0.007 & 0.007 & 0.007 & 0.007 & 0.007 & 1.1 & 0.011 & 0.011 & 0.011 & 0.011 & 0.011 \\
\hline 19 & Thyroid cancer & 0.9 & 0.009 & 0.007 & 0.007 & 0.007 & 0.007 & 3.4 & 0.034 & 0.027 & 0.025 & 0.024 & 0.027 \\
\hline 20 & Hodgkin lymphoma & 0.7 & 0.007 & 0.005 & 0.005 & 0.005 & 0.005 & 0.8 & 0.008 & 0.006 & 0.006 & 0.006 & 0.006 \\
\hline & Total & 100.1 & 1.001 & 0.977 & 1.029 & 1.047 & 1.200 & 99.3 & 0.993 & 0.974 & 1.077 & 1.100 & 1.177 \\
\hline
\end{tabular}

Table 3: Risk contribution for twenty cancer types reported to be possibly associated with alcohol

drinking in the Western World in both men and women per alcohol consumption category 
The sigmoidal dose response model with logEC50 value was used to evaluate the impact of alcohol consumption (in $g$ alcohol/day) on cancer risk for the various types of cancer as well as for overall cancer (Table 4). Overall cancer risk showed a lower logEC50 value (value at which $50 \%$ of the potential maximum effect is obtained) for women ( $22.3 \mathrm{~g}$ ) than for men ( $45.5 \mathrm{~g}$ ), suggesting that women are more sensitive to alcohol in terms of their overall cancer risk. The logEC50 was relatively low (at about $8 \mathrm{~g}$ alcohol/day) in cancers such as breast cancer and prostate cancer suggesting that these cancers may be more sensitive to alcohol consumption than other cancers. Other cancers like Hodgkin and non-Hodgkin may be less sensitive or may even have some lower risk associated with alcohol consumption. The model did not show an increase or a decrease in cancer risk for two cancer types (glioma (brain cancer) and gall bladder cancer) and the model could not fit the data for three cancer types (lung cancer, multiple myeloma and leukemia) due to the absence of an asymptotic level. A parabolic function better described the associations for those three cancer types: lung cancer $\left(\mathrm{R}^{2}: 0.83\right.$ for both men and women), leukemia $\left(\mathrm{R}^{2}: 0.16\right.$ for men and 0.20 for women) and multiple myeloma ( $\mathrm{R}^{2}: 0.97$ for both men and women).

\begin{tabular}{|c|c|c|c|c|c|c|}
\hline & & & \multicolumn{2}{|c|}{ Men } & \multicolumn{2}{c|}{ Women } \\
\hline & Cancer type & Direction & logEC50 & R2 & logEC50 & R2 \\
\hline 1 & Breast cancer & up & --- & --- & 9.6 & 0.97 \\
\hline 2 & Prostate cancer & up & 8.2 & 0.90 & --- & --- \\
\hline 3 & Colorectal cancer & up & 22.8 & 0.94 & 22.8 & 0.94 \\
\hline 4 & Lung cancer & down & N.A.+ & N.A.+ & N.A.+ & N.A.+ \\
\hline 5 & Bladder cancer & down & 22.5 & 1.00 & 33.8 & 1.00 \\
\hline 6 & Renal cancer & down & 8.7 & 0.92 & 22.4 & 0.90 \\
\hline 7 & Gastric cancer & up & 21.9 & 0.93 & 9.1 & 0.94 \\
\hline 8 & Ovarian cancer & up & --- & --- & 9.5 & 0.95 \\
\hline 9 & Non-Hodgkin lym- & down & 8.2 & 0.97 & 8.2 & 0.97 \\
\hline 10 & Shoma & up & 22.7 & 0.97 & 22.7 & 0.98 \\
\hline 11 & Pancreas cancer & up & 37.3 & 0.97 & 37.4 & 0.84 \\
\hline 12 & Leukemia & down & N.A.+ & N.A.+ & N.A.+ & N.A.+ \\
\hline 13 & Liver cancer & up & 52.7 & 0.96 & 54.4 & 0.95 \\
\hline 14 & Esophageal cancer & up & 45.6 & 0.93 & 45.6 & 0.93 \\
\hline 15 & Larynx cancer & up & 45.5 & 0.89 & 45.8 & 0.96 \\
\hline 16 & Glioma, brain cancer & absent\& & N.A.+ & N.A.+ & N.A.+ & N.A.+ \\
\hline 17 & Multiple myeloma & down & N.A.+ & N.A.+ & N.A.+ & N.A.+ \\
\hline 18 & Gall bladder cancer & absent\& & N.A.+ & N.A.+ & N.A.+ & N.A.+ \\
\hline 19 & Thyroid cancer & down & 2.9 & N.A. & 8.1 & 0.92 \\
\hline 20 & Hodgkin lymphoma & down & 2.9 & 1.00 & 2.9 & 1.00 \\
\hline & Overall & up & 45.5 & 0.95 & 22.3 & 0.87 \\
\hline
\end{tabular}

* up means increased risk; down means decreased risk with increasing alcohol consumption.

\& absent: no clear increase or decrease in cancer risk observed

${ }^{+}$not applicable: the (relative lack of) data did not allow a model fit

logEC50: alcohol consumption level (g/day) at which $50 \%$ of the effect is reached

$\mathrm{R}^{2}$ : variation explained by the model

Table 4: The sigmoidal dose response model (Hill equation) parameters for the twenty most

common cancer types and overall cancer risk

Figure 2 shows the overall cancer risk of the 20 most common cancers in the Western world associated with alcohol consumption based on the data fitted in to the sigmoidal dose response model. The model outcome in men was $1.00+0.20 /\left(1+10^{(45.53-\mathrm{x})}\right)$, whereas in women it was $0.98+0.16 /\left(1+10^{(22.31-\mathrm{x})}\right)$, demonstrating an increased risk at lower alcohol consumption for women as compared to men; cancer risk increases in women at a daily consumption level that is about half the level observed for men ( $22 \mathrm{~g}$ versus $46 \mathrm{~g})$.

\section{Discussion}

In the present meta-analysis we found that overall cancer risk in the 20 most common types of cancer in the western world are slightly lower in the first drinking category (2.5-14.9 g alcohol/day) and slightly higher in the next two drinking categories (15 up to $60 \mathrm{~g}$ alcohol/day) as compared to non-drinking. Overall cancer risk only increased substantially when drinking $60 \mathrm{~g}$ or more alcohol/day. Women appeared to be more sensitive for an increased risk: the dose of alcohol to acquire half of the risk increase is $22 \mathrm{~g} /$ day for women and $46 \mathrm{~g} /$ day for men. 

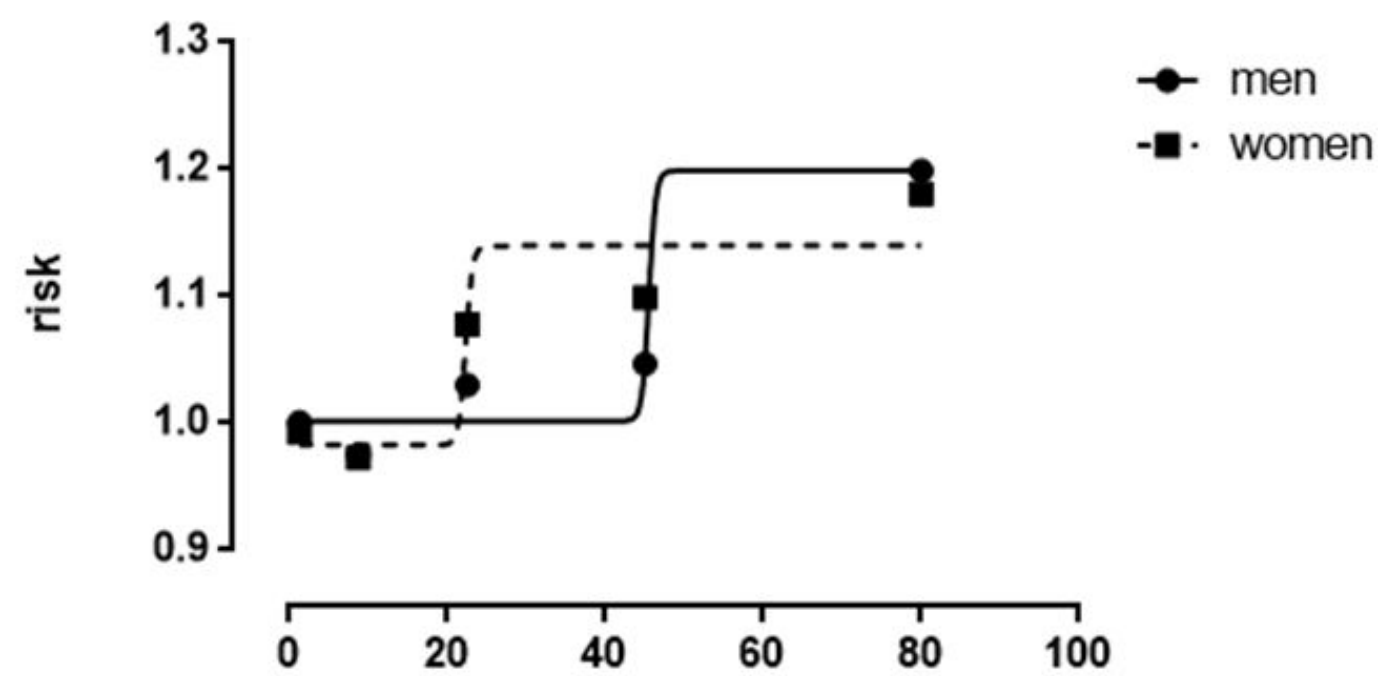

\section{alcohol (g/day)}

Symbols represent the mean value per alcohol category. The solid line represents the men and the dotted line the women. Figure 2: The sigmoidal dose response model for cancer risk of the 20 most common cancers in the western world per gender associated with alcohol consumption

The relation between alcohol consumption and overall cancer incidence is complex, due to the diversity of the associations for the various types of cancers. This study was initiated to try and quantify the effect of alcohol consumption on overall cancer incidence in the Western world when drinking in moderation. Drinking alcohol in moderation is defined in most Western countries as consuming between 2.5 and $15 \mathrm{~g}$ alcohol/day for women and between 2.5 and $30 \mathrm{~g}$ alcohol/day for men. We showed that drinking in moderation has no substantial impact on overall cancer risk. Moderate alcohol consumption as such may therefore be considered as a less important lifestyle factor for overall cancer risk modulation as compared to other lifestyle factors, such as smoking and obesity. In contrast, heavy drinking, viz. more than $60 \mathrm{~g}$ alcohol/day is associated with an increased incidence for most types of cancer investigated in the present study. It is noteworthy that the association with light and moderate alcohol consumption is negative for cancers such as (non)-Hodgkin, leukemia, lung and renal cancer, but positive for other cancers, such as breast, prostate, ovarian, gastric and colorectal cancer.

Several assumptions were made during our study that may have affected the outcome. The most recent meta-analyses, covering the largest number of studies including cohort studies, were selected for inclusion in the present study. We did not evaluate indepth quality criteria of these meta-analyses assuming that peer review would yield satisfactory quality. A number of aspects may, however, need to be taken into account. These aspects include the role of confounding factors, risks for specific subgroups, drinking categories choice and a potential role for underreporting.

In some associations, a strong confounding effect exists, which may be difficult to correct for. This may for instance be the case for lung cancer and smoking; smoking is a very strong risk factor for lung cancer. Therefore, we chose a meta-analysis that focused on non-smokers only. The RRs observed in these never smokers were, however, not very different from those described in a mixed population as pointed out by the authors [23,24]. Overall, it is a very difficult to correct for confounding, but by choosing meta-analyses applying fully adjusted models, the best possible estimate for the association between alcohol consumption and the specific type of cancer may have been obtained.

As far as subgroup risks are concerned it is known that for instance breast cancer risk may depend on menopausal status and genetic background $[20,25,26]$. This means that alcohol consumption may contribute more to the breast cancer risk for a specific subgroup than in others.

Although we selected those meta-analyses that allowed for the extraction of data that would fit our drinking categories, this was not a complete match in all cases. Also, the choice of the control group could have affected the outcome. A control group consisting of teetotalers combined with very rare or occasional alcohol consumers, originates from the discussion on teetotalers possibly being a debatable control [27]. Occasional alcohol consumers may differ from teetotalers in some confounders like disease history or other confounders that cannot be corrected for. In order to try and minimize such bias a combined control group was used. Also, various cancer types had various associations: some were J-shaped others were linear-shaped or logarithmic-shaped. These different associations were found in meta-analyses covering numerous cancer types [16,21]. Therefore, it seems best to use a combination control group as has been currently used in a large number of meta-analyses. 
Underreporting may play a role in the interpretation of the alcohol cancer risk association. Klatsky et al explored the role of inferred underreporting among light-moderate drinkers on the association between alcohol intake and cancer risk [28]. This study suggested that the increased risk of cancer among light-moderate drinkers occurred mainly in those suspected of underreporting, whereas the increased risk was not observed in those not suspected of underreporting or in those of unsure reporting status [28]. We have used the relative risks reported in the various meta-analyses assuming that the chance of acquiring various cancers would be equally distributed over the drinking categories. This assumption, however, may not be entirely correct. An alternative method used is to correlate the overall incidence to the average relative risk observed and derive the relative risks for the other drinking categories from this average. We chose not to use this method since the final outcome would most likely not be substantially different as compared to the current results. Also, the average drinking levels corresponding to the average relative risk is unknown. Therefore, we chose to use the relative risks as they were calculated for the large groups of people included in the studies of these meta-analyses.

A number of meta-analyses selected for this study distinguished in types of alcoholic beverages, such as Duell and Fagherazzi amongst others. We did not make this distinction in our analysis for various reasons [20,29]. Firstly, such a beverage distinction was not available for all cancer types studied. Secondly, we believe that a wine drinker may differ in many aspects from a beer drinker, e.g. a wine drinker would eat a more healthy diet [30]. A comparison between the various alcoholic beverages would be confounded by too many factors that were not controlled for in many of the studies used in the present analysis. Also, the large majority of alcohol consumers does not have a preference for one type of alcoholic beverage, but consume several. Since it is very complex to unravel the contribution of each of those beverages, we did not try to make separate analyses for the various alcoholic beverages.

The results of this overall estimate were made based on meta-analyses that mainly used data collected in Europe, US and other Western world countries. It has been described that some cancers occur in higher or lower incidences in other parts of the world, therefore regional differences exist. For instance, breast cancer appears to be not associated with alcohol consumption at all in Asian countries [10,31]. Also, lifestyle may differ and therefore our conclusions cannot be generalized to the non-Western world.

In the present study, a novel approach was applied to associate daily alcohol intake and cancer risk. Various models were evaluated; the best fit was obtained using the sigmoidal dose response model using the logEC50 value. The model showed an increase in overall cancer risk at increasing daily alcohol consumption with women being more vulnerable than men. This is in agreement with a higher sensitivity for alcohol use disorder and its associated diseases such as alcohol-related liver disease and cardiomyopathy in women than in men [12]. This approach, however, is limited by the number of observations, viz. the number of alcohol drinking categories. More research is needed in which alcohol consumption may be divided in more and smaller drinking categories allowing for models with a better fit. These logEC50 values also show that there is a difference in the risk of alcohol consumption on overall cancer as compared to an individual type of cancer. The outcome on overall cancer is a summation of all positive and negative associations. Therefore, it would be important to publicize not only the overall association of alcohol with cancer but also its associations with each individual type of cancer.

We were interested to apply an approach that would allow us to provide an overall risk estimate for consuming alcohol lightly and in moderation. A recent study by Choi et al. reached very similar conclusions as reported here: very light to light alcohol drinking $(<12.5 \mathrm{~g}$ alcohol/day) was not associated with an increased risk of the most common cancer types. These authors focus on several methodological flaws in meta-analyses reporting positive associations between light and moderate alcohol consumption and specific cancer types [9].

The new aspect of this analysis is the integration of data on most frequently occurring types of cancers to estimate the overall impact of alcohol consumption on total cancer incidence. This is important since alcohol consumption is promoted as one of the most important modifiable lifestyle factors contributing to overall cancer incidence being a group 1 carcinogen [32,33]. The present study suggests that alcohol, when consumed in moderation, may not be a very important factor to modify for cancer prevention in general. For cancer prevention, it may be worthwhile to focus more on other modifiable lifestyle factors contributing significantly to overall cancer incidence. These may include smoking or focus on other modifiable life style factors that protect better to overall cancer incidence like for instance physical activity [34,35].

The data presented suggest that light and moderate alcohol consumption (up to $15 \mathrm{~g}$ alcohol/day) is not associated with the incidence of the 20 most common cancer types in the Western world in contrast to higher consumption [36-51].

\section{Acknowledgements}

This work was supported by Knowledge Institute Beer (KIB). KIB had no role in the study design, data collection and analysis, decision to publish nor in the preparation of the manuscript. The authors declare no conflict of interest.

\section{References}

1. Siegel RL, Miller KD, Jemal A (2017) Cancer Statistics, 2017. CA Cancer J Clin 67: 7-30.

2. Malvezzi M, Carioli G, Bertuccio P, Rosso T, Boffetta P, et al. (2016) European cancer mortality predictions for the year 2016 with focus on leukaemias. Ann Oncol 27: 725-31. 
3. Arteaga CL, Adamson PC, Engelman JA, Foti M, Gaynor RB, et al. (2014) AACR Cancer Progress Report 2014. Clin Cancer Res 20: S1-S112.

4. Kerr J, Anderson C, Lippman SM (2017) Physical activity, sedentary behaviour, diet, and cancer: an update and emerging new evidence. Lancet Oncol 18: e457e71.

5. Consumption of alcoholic beverages (2012) IARC monographs on the evaluation of carcinogenic risks to humans.100 E(Pt E): 373-499.

6. Zhou Y, Zheng J, Li S, Zhou T, Zhang P, et al (2016) Alcoholic beverage consumption and chronic diseases. Int J Environ Res Public Health 13:522.

7. de Gaetano G, Costanzo S, Di Castelnuovo A, Badimon L, Bejko D, et al. (2016) Effects of moderate beer consumption on health and disease: A consensus document. Nutr Metab Cardiovasc Dis 26: 443-67.

8. Cao Y, Willett WC, Rimm EB, Stampfer MJ, Giovannucci EL (2015) Light to moderate intake of alcohol, drinking patterns, and risk of cancer: results from two prospective US cohort studies. BMJ 351: h4238.

9. Choi YJ, Myung SK, Lee JH (2018) Light alcohol drinking and risk of cancer: a meta-analysis of cohort studies. Cancer Res Treat 50: 474-87.

10. Bagnardi V, Rota M, Botteri E, Tramacere I, Islami F, et al. (2013) Light alcohol drinking and cancer: a meta-analysis. Ann Oncol 24: 301-8.

11. Parry CD, Patra J, Rehm J (2011) Alcohol consumption and non-communicable diseases: epidemiology and policy implications. Addiction 106: 1718-24.

12. European Cancer Information System (2018) EUCAN, Cancer, UK.

13. Siegel S, Castellan NJ (1988) Nonparametric Statistics for the Behavioral Sciences. McGraw-Hill Publishing Co, USA.

14. Zhang HZ, Jin GF, Shen HB (2012) Epidemiologic differences in esophageal cancer between Asian and Western populations. Chin J Cancer 31: 281-6.

15. Torre LA, Siegel RL, Ward EM, Jemal A (2016) Global cancer incidence and mortality rates and trends-an update. Cancer Epidemiol Biomarkers Prev 25:16-27. 16. Bagnardi V, Rota M, Botteri E, Tramacere I, Islami F, et al. (2015) Alcohol consumption and site-specific cancer risk: a comprehensive dose-response metaanalysis. Br J Cancer 112: 580-93.

17. Hendriks HF, Stockley CS, Calame W (2015) The 2015 Dutch food-based dietary guidelines on alcohol consumption - a critical review. J Nutr Health Sci 5: 303.

18. Gadagkar SR, Call GB (2015) Computational tools for fitting the Hill equation to dose-response curves. J Pharmacol Toxicol Methods 71: 68-76.

19. Smith-Warner SA, Spiegelman D, Yaun SS, van den Brandt PA, Folsom AR, et al (1998) Alcohol and breast cancer in women: a pooled analysis of cohort studies. JAMA 279: 535-40.

20. Fagherazzi G, Vilier A, Boutron-Ruault MC, Mesrine S, Clavel-Chapelon F (2015) Alcohol consumption and breast cancer risk subtypes in the E3N-EPIC cohort. Eur J Cancer Prev 24: 209-14.

21. Jayasekara H, MacInnis RJ, Room R, English DR (2016) Long-term alcohol consumption and breast, upper aero-digestive tract and colorectal cancer risk: a systematic review and meta-analysis. Alcohol Alcohol 51: 315-30.

22. Choo SP, Tan WL, Goh BK, Tai WM, Zhu AX (2016) Comparison of hepatocellular carcinoma in Eastern versus Western populations. Cancer.

23. Fehringer G, Brenner DR, Zhang ZF, Lee YA, Matsuo K, et al. (2017) Alcohol and lung cancer risk among never smokers: A pooled analysis from the international lung cancer consortium and the SYNERGY study. Int J Cancer 140: 1976-84.

24. Troche JR, Mayne ST, Freedman ND, Shebl FM, Abnet CC (2016) The association between alcohol consumption and lung carcinoma by histological subtype. Am J Epidemiol 183: 110-21.

25. Dartois L, Fagherazzi G, Baglietto L, Boutron-Ruault MC, Delaloge S, et al. (2016) Proportion of premenopausal and postmenopausal breast cancers attributable to known risk factors: Estimates from the E3N-EPIC cohort. Int J Cancer 138: 2415-27.

26. Tamimi RM, Spiegelman D, Smith-Warner SA, Wang M, Pazaris M, et al. (2016) Population attributable risk of modifiable and nonmodifiable breast cancer risk factors in postmenopausal breast cancer. Am J Epidemiol 184: 884-93.

27. Stockwell T, Zhao J, Panwar S, Roemer A, Naimi T, et al. (2016) Do "moderate" drinkers have reduced mortality risk? A systematic review and meta-analysis of alcohol consumption and all-cause mortality. J Stud Alc Drugs 77: 185-98.

28. Klatsky AL, Udaltsova N, Li Y, Baer D, Nicole Tran H, et al. (2014) Moderate alcohol intake and cancer: the role of underreporting. Cancer Causes Control 25: 693-9.

29. Duell EJ, Travier N, Lujan-Barroso L, Clavel-Chapelon F, Boutron-Ruault MC, et al. (2011) Alcohol consumption and gastric cancer risk in the European Prospective Investigation into Cancer and Nutrition (EPIC) cohort. Am J Clin Nutr 94: 1266-75.

30. Tjonneland A, Gronbaek M, Stripp C, Overvad K (1999) Wine intake and diet in a random sample of 48763 Danish men and women. Am J Clin Nutr 69: 49-54.

31. Li Y, Yang H, Cao J (2011) Association between alcohol consumption and cancers in the Chinese population - a systematic review and meta-analysis. PLOS ONE 6: e18776.

32. Khan N, Afaq F, Mukhtar H (2010) Lifestyle as risk factor for cancer: Evidence from human studies. Cancer Lett 293: 133-43.

33. IARC (2012) Monographs on the evaluation of carcinogenic risks to humans: consumption of alcoholic beverages, Lyon, France.

34. Arnold M, Pandeya N, Byrnes G, Renehan PAG, Stevens GA, et al. (2015) Global burden of cancer attributable to high body-mass index in 2012: a populationbased study. Lancet Oncol 16: 36-46.

35. Friedenreich CM, Neilson HK, Lynch BM (2010) State of the epidemiological evidence on physical activity and cancer prevention. Eur J Cancer 46: $2593-604$. 36. Vieira AR, Abar L, Chan D, Vingeliene S, Polemiti E, et al. (2017) Foods and beverages and colorectal cancer risk: a systematic review and meta-analysis of cohort studies, an update of the evidence of the WCRF-AICR Continuous Update Project. Ann Oncol 28: 1788-802.

37. Pelucchi C, Galeone C, Tramacere I, Bagnardi V, Negri E, et al. (2012) Alcohol drinking and bladder cancer risk: a meta-analysis. Ann Oncol $23: 1586-93$.

38. Xu X, Zhu Y, Zheng X, Xie L (2015) Does beer, wine or liquor consumption correlate with the risk of renal cell carcinoma? A dose-response meta-analysis of prospective cohort studies. Oncotarget 6: 13347-58.

39. Fang X, Wei J, He X, An P, Wang H, et al. (2015) Landscape of dietary factors associated with risk of gastric cancer: A systematic review and dose-response meta-analysis of prospective cohort studies. Eur J Cancer 51: 2820-32.

40. Yan-Hong H, Jing L, Hong L, Shan-Shan H, Yan L, et al. (2015) Association between alcohol consumption and the risk of ovarian cancer: a meta-analysis of prospective observational studies. BMC Pub Health 15: 223.

41. Tramacere I, Pelucchi C, Bonifazi M, Bagnardi V, Rota M, et al. (2012) Alcohol drinking and non-Hodgkin lymphoma risk: a systematic review and a metaanalysis. Ann Oncol 23: 2791-8. 
42. Rota M, Pasquali E, Bellocco R, Bagnardi V, Scotti L, et al. (2014) Alcohol drinking and cutaneous melanoma risk: a systematic review and dose-risk metaanalysis. Br J Dermatol 170: 1021-8.

43. Wang YT, Gou YW, Jin WW, Xiao M, Fang HY (2016) Association between alcohol intake and the risk of pancreatic cancer: a dose-response meta-analysis of cohort studies. BMC Cancer 16: 212.

44. Rota M, Porta L, Pelucchi C, Negri E, Bagnardi V, et al. (2014) Alcohol drinking and risk of leukemia-a systematic review and meta-analysis of the dose-risk relation. Cancer Epidemiol 38: 339-45.

45. Islami F, Fedirko V, Tramacere I, Bagnardi V, Jenab M, et al. (2011) Alcohol drinking and esophageal squamous cell carcinoma with focus on light-drinkers and never-smokers: a systematic review and meta-analysis. Int J Cancer 129: 2473-84.

46. Islami F, Tramacere I, Rota M, Bagnardi V, Fedirko V, et al. (2010) Alcohol drinking and laryngeal cancer: overall and dose-risk relation - a systematic review and meta-analysis. Oral Oncol 46: 802-10.

47. Qi ZY, Shao C, Yang C, Wang Z, Hui GZ (2104) Alcohol consumption and risk of glioma: a meta-analysis of 19 observational studies. Nutrients 6: 504-16.

48. Galeone C, Malerba S, Rota M, Bagnardi V, Negri E, et al. A meta-analysis of alcohol consumption and the risk of brain tumours. Ann Oncol 24: 514-23.

49. Rota M, Porta L, Pelucchi C, Negri E, Bagnardi V, et al. (2014) Alcohol drinking and multiple myeloma risk - a systematic review and meta-analysis of the doserisk relationship. Eur J Cancer Prev 23: 113-21.

50. Hong SH, Myung SK, Kim HS (2017) Alcohol intake and risk of thyroid cancer: A meta-analysis of observational studies. Cancer Res Treat 49 : 534-47.

51. Tramacere I, Pelucchi C, Bonifazi M, Bagnardi V, Rota M, et al. (2012) A meta-analysis on alcohol drinking and the risk of Hodgkin lymphoma. Eur J Cancer Prev 21: 268-73. 